\title{
Parallel Numerical Creation of 2-parametric Bifurcation Diagram of Nonlinear Oscillators
}

\author{
F. Hajdu \\ Széchenyi István University, Faculty of Mechanical Engineering, Informatics \\ and Electrical Engineering, Department of Mechatronics and Machine Design \\ Egyetem tér 1., 9026 Győr, Hungary \\ e-mail: hajdfl@sze.hu
}

Abstract: This paper presents the numerical creation of 2-parametric bifurcation diagrams of nonlinear oscillators with a simple iterative algorithm, which can be easily parallelized. The parallel algorithm was tested with two simple well-known nonlinear oscillators, the Van der Pol oscillator and the Duffing-Holmes oscillator. It was examined how the resolution (number of iterations) affects the speedup and the efficiency. The test results show that a relative good speed up with a good efficiency could be achieved even using a simple desktop.

Keywords: parallel computing, bifurcation diagram, numerical analysis, nonlinear oscillator, Maple

\section{Introduction}

Bifurcation diagram is a very useful tool to study nonlinear dynamical systems. It shows the systems behaviour as a system parameter is varied [1]. In most cases the bifurcation diagram of a single parameter is calculated [2]-[9], but a system can have more bifurcation parameters [10]. In order to examine the effect of more bifurcation parameters at the same time a 2 parametric or 3D bifurcation diagram can be created [11]. However constructing a detailed 3D bifurcation diagram can be time consuming even in case of simple systems, as a lot of numerical calculation is necessary. These calculations can be fastened using more processor cores [12]. The aim of this research was to create 3D bifurcation diagrams within a reasonable time using simple PC-s.

The paper first presents some examples using 3D bifurcation diagrams found in the literature, then the sequential and parallel numerical creation of $3 \mathrm{D}$ bifurcation 
diagrams are described, which is followed by some test results. The paper concludes with further development tasks.

\section{Creation of bifurcation diagram with more parameters}

In the literature some examples of simulations using 3D bifurcation diagrams and methods for calculating and visualizing bifurcation surfaces of more parameters can be found.

In [11], the creation of the 3D bifurcation diagram is presented with a predatorprey model. The bifurcation points are calculated with the Newton-Raphson method. Then the surface diagram is created with triangulation.

In [13], the use of different 3D bifurcation diagram is presented with population model examples, like ecosystem models and predator-prey models. A 3D bifurcation diagram with 2 state variables and a bifurcation parameter is used to determine the stability of the systems. 3D bifurcation diagrams with 3 different parameters are calculated to examine the equilibrium points and the type of bifurcations.

Reference [14] proposes an efficient algorithm to calculate and visualize three dimensional bifurcation surfaces. The algorithm was tested with a socio-economic model, a metabolic network and a food-chain model.

In [15], an analytical method based on resultant is presented to calculate bifurcation surfaces. The presented method is suitable for medium size systems and can be used to detect Hopt bifurcations and some higher codimension bifurcations too.

More parametric bifurcation diagrams have been used to study electrical systems. In [16], ferroresonance of power systems is predicted with 3D bifurcation diagrams. The 3D bifurcation diagram is produced with creating as many $2 \mathrm{D}$ bifurcation diagrams as the second parameter range. The 2D bifurcation diagram is created with repeating time-domain simulations followed by frequency-domain sampling of the same output to determine its periodicity. The creation of bifurcation diagram was speed up using a 50 core parallel computer [17].

In [18], the bifurcation analysis of a permanent magnet synchronous motor was achieved using 2D bifurcation curves and 3D bifurcation surfaces. The bifurcation surfaces were created from bifurcation curves calculated with different values of the third parameter.

In [19], the 3D bifurcation diagram of a simple electrical system with a nonlinear Tunnel diode is created using Maple's implicitplot command. 
2D and 3D bifurcation diagrams can be used to examine biochemical reactions too. In [20], stable states of a protometabolic network are studied with $2 \mathrm{D}$ and 3D bifurcation diagrams.

The creation of 3D bifurcation diagram can be very time consuming [17]. The aim of this research was to expand a fast and efficient algorithm used to calculate $2 \mathrm{D}$ bifurcation diagrams in a way to create $3 \mathrm{D}$ bifurcation diagrams even on simple PC-s within a reasonable time.

\subsection{The algorithm to create the $3 D$ bifurcation diagram}

The 2D bifurcation diagram can be created with a simple iterative algorithm based on the algorithm described in [21]. The flowchart of the algorithm can be seen in Figure 1.

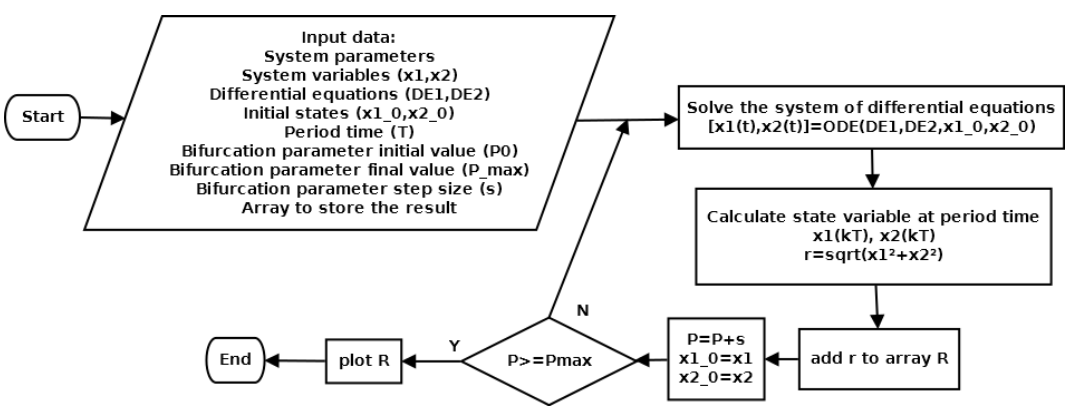

Figure 1. Algorithm to create the bifurcation diagram

The 3D bifurcation diagram can be created if the 2D bifurcation diagrams are calculated with every value of a different bifurcation parameter and the results are displayed in a 3D plot (Figure 2). 


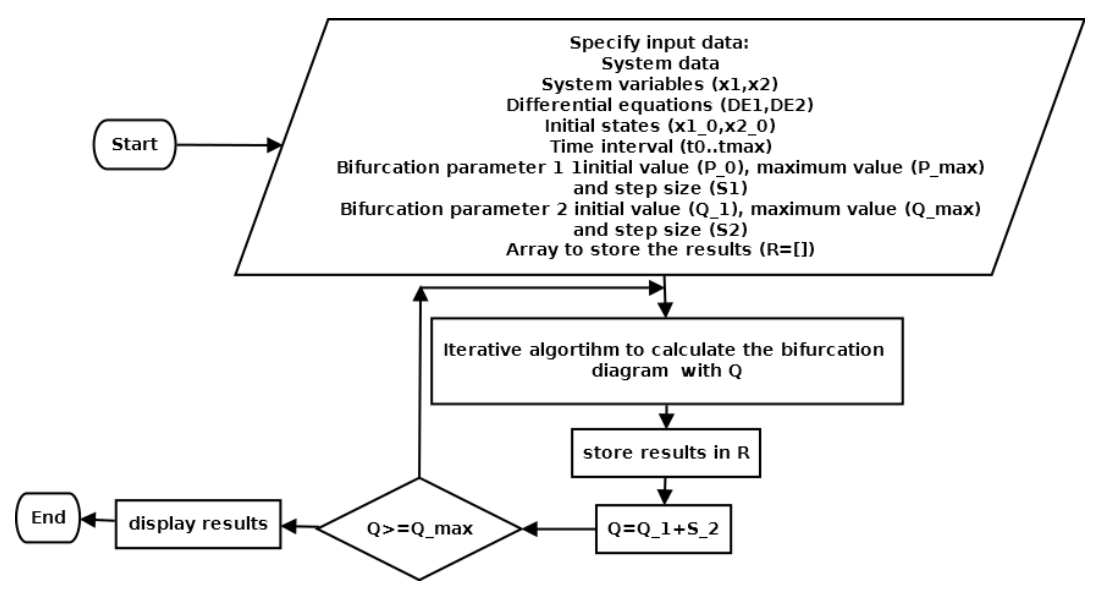

Figure 2. Algorithm to create the $3 D$ bifurcation diagram

In this study the numerical 3D bifurcation diagram of the forced Duffing-Holmes and the forced Van der Pol oscillator is created. Maple was used as it is a powerful tool for both symbolic [22] and numerical calculations[23]. The equation of the Duffing-Holmes oscillator is:

$$
\frac{d^{2}}{d t^{2}} x(t)+\delta \frac{d}{d t} x(t)+\beta x(t)+\alpha x(t)^{3}=\gamma \cos (\omega t)
$$

In this study parameters $\alpha=1, \beta=-1$ and $\omega=1$ were chosen as constants and $\delta$ and $\gamma$ are the bifurcation parameters. The bifurcation diagram can be seen in Figure 3 .
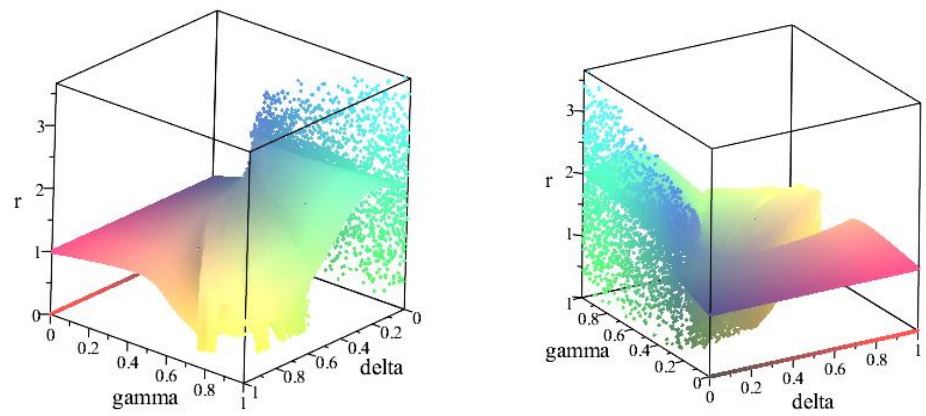

Figure 3. 3D bifurcation diagram of the Duffing-Holmes oscillator 
From the diagram the bifurcation regions can be observed, which are:

- $\gamma=0$

- $\delta=0$

- the region between $\gamma=4 / 3 \times \delta+0.2$ and $\gamma=0.6 \times \delta+0.2$

To test the accuracy of the bifurcation diagram some Poincare sections were created, which can be seen in Figure 4.
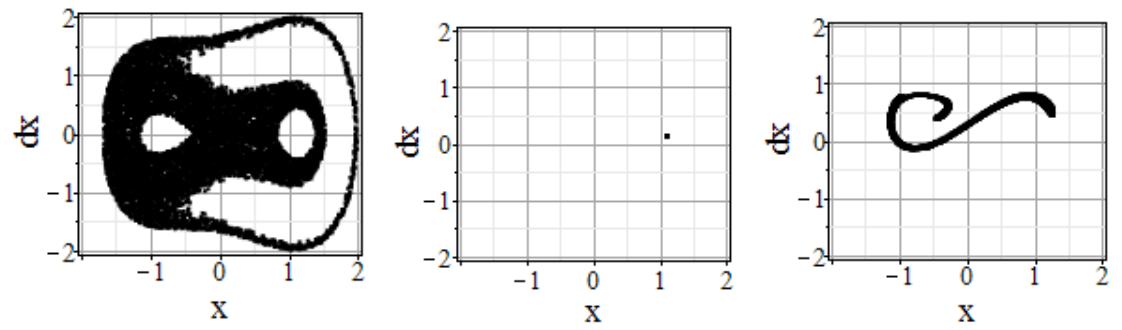

Figure 4. Poincaré sections of the Duffing-Holmes oscillator ( $\gamma=0.1$ and $\delta=0$, $\gamma=0.2$ and $\delta=0.8, \gamma=0.6$ and $\delta=0.5$ )

It can be seen, that the Poincaré sections also show the chaotic behaviour at the same parameter values as the bifurcation diagrams. When $\gamma=0.1$ and $\delta=0$ and $\gamma=0.6$ and $\delta=0.5$ there is a chaotic oscillation and in case of $\gamma=0.2$ and $\delta=0.8$ there is a harmonic oscillation.

The equation of the forced Van der Pol oscillator is:

$$
\frac{d^{2}}{d t^{2}} x(t)=\mu\left(1-x(t)^{2}\right) \frac{d}{d t} x(t)-x(t)+A \cos (\omega t)
$$

In this study $\omega=2 \pi / 10$ and $\mu$ and $A$ are the bifurcation parameters. The $3 \mathrm{D}$ bifurcation diagram can be seen in Figure 5. 

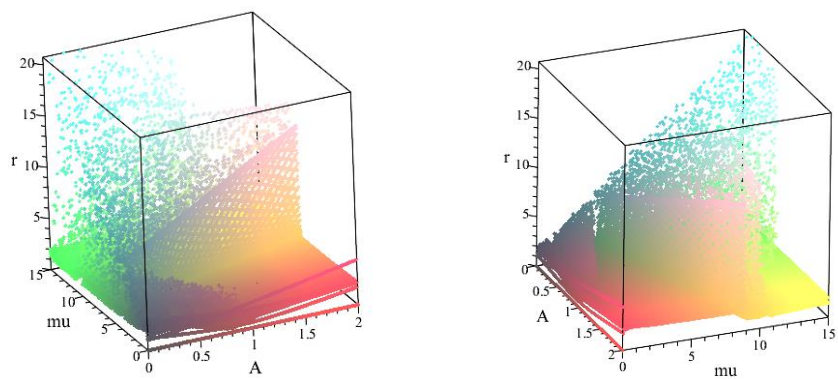

Figure 5. 3D bifurcation diagram of the Van der Pol oscillator

From the diagram the bifurcation regions can be observed, which are:

- the region $\mu<=10 \times \mathrm{A}$

- between $\mu=2.5 \times \mathrm{A}, \mathrm{A}=[1.2-2]$ and $\mu=10, \mathrm{~A}=[0,1.2]$ and $\mu=15, \mathrm{~A}=0$

If $\mu=0$ there is a subharmonic oscillation. Some Poincaré sections were created to test the accuracy of the bifurcation diagram with a different system too (Figure 6).
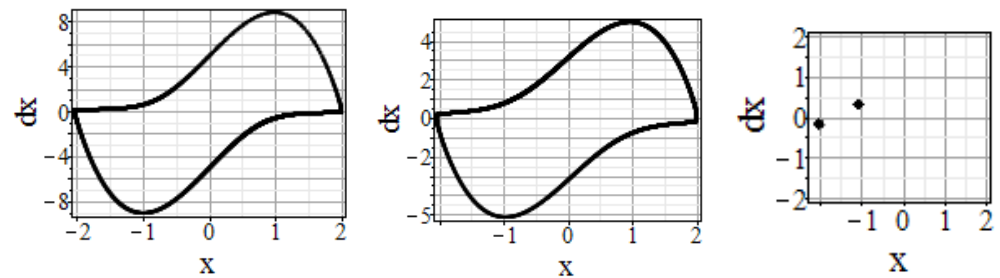

Figure 6. Poincaré sections of the Van der Pol oscillator ( $\mu=6$ and $A=0.5, \mu=3$ and $A=0.2$ and $\mu=10$ and $A=1.5$ )

It can be seen that similarly the previous system the Poincaré sections shows the system behaviour according to the $3 \mathrm{D}$ bifurcation diagram: when $\mu=6, \mathrm{~A}=0.5$ and $\mu=3$ and $A=0.2$ there is a chaotic limit cycle and when $\mu=10$ and $A=1.5$ there is a subharmonic oscillation.

As the same iterative algorithm is used with every values of the bifurcation parameters this task can be easily parallelized. 


\subsection{Parallel algorithm}

The numerical creation of the 2 parametric bifurcation diagram can be parallelized with a similar algorithm presented in [24]. For parallelization Maple's Grid programming model was used. There is a master node to supervise the slave nodes and to collect the results. The slave nodes calculate the data for the bifurcation diagram for each bifurcation parameter and send the results to the master node. The flow chart of the parallel algorithm can be seen in Figure 7 and the Pseudo code of the Maple program can be seen in Figure 8.

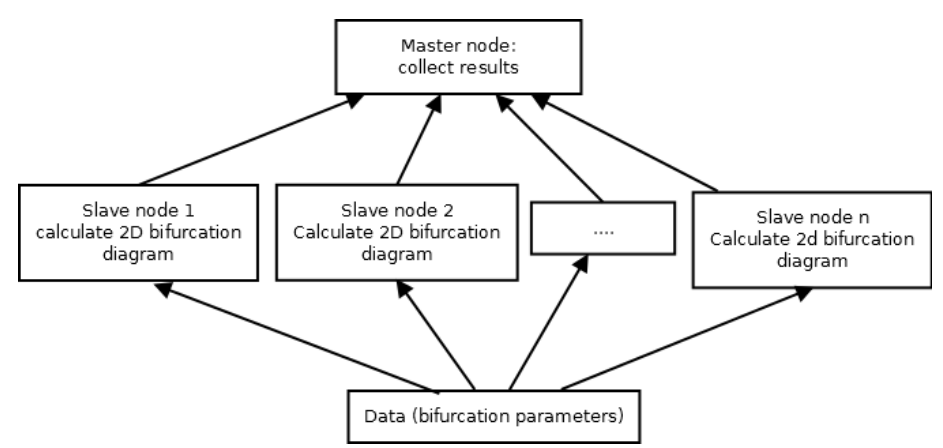

Figure 7. Flow chart of the Grid Programming model (SIMD parallel algorithm) 


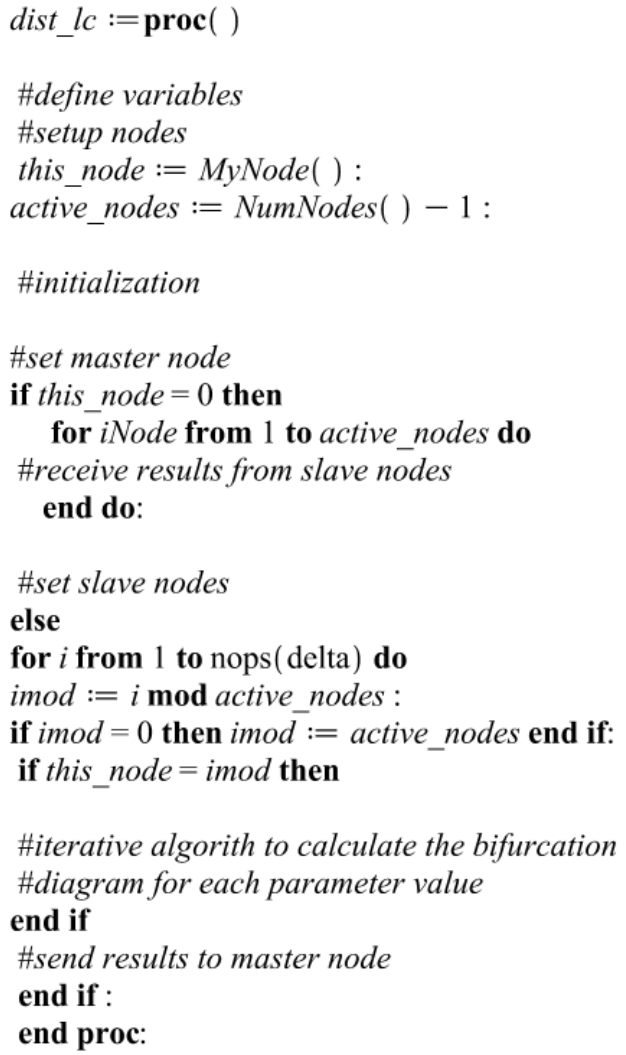

Figure 8. Pseudo code of the parallel Maple program

\section{Creation of bifurcation diagrams with more parameters}

The tests were carried out on a PC with an Intel ${ }^{\circledR}$ Core ${ }^{\mathrm{TM}}$ i5-4460 Processor 3.2 $\mathrm{GHz}$ and $16 \mathrm{~GB}$ RAM. The processor has 4 cores

The speedup, the efficiency and the overhead of the program were calculated with the following formulas [25]-[27]: 


$$
\begin{gathered}
S=\frac{T_{s}}{T_{p}} \\
E=\frac{S}{n} \\
O=\frac{T_{p}-T_{u}}{T_{p}}
\end{gathered}
$$

where $T_{s}$ is the sequential calculation time, $T_{p}$ is the parallel running time, $T_{u}$ is the time of the useful work (calculation time of a $2 \mathrm{D}$ bifurcation diagram), $\mathrm{n}$ is the number of cores.

To change the resolution of the bifurcation diagram the number of iteration was varied for both parameters:

$$
\text { resolution }=\frac{1}{N}
$$

where $\mathrm{N}$ is the number of iterations. The number of iterations for each resolution can be seen in Table 1. G is parameter of the iterative algorithm to create a single bifurcation diagram $(\gamma$ and $\mu)$ and $D$ is the parameter for the number of bifurcation diagrams in $3 \mathrm{D}(\delta$ and $\mathrm{A})$.

Table 1. Number of iterations as resolution of the bifurcation parameters ( $G$ and D) is changed

\begin{tabular}{|c|c|c|c|c|c|c|c|c|}
\hline & $\mathbf{D}$ & $\mathbf{1 1}$ & $\mathbf{2 6}$ & $\mathbf{5 1}$ & $\mathbf{1 0 1}$ & $\mathbf{2 5 1}$ & $\mathbf{5 0 1}$ & $\mathbf{1 0 0 1}$ \\
\hline $\mathbf{G}$ & & & & & & & & \\
\hline $\mathbf{1 1}$ & & 121 & 286 & 561 & 1111 & 2761 & 5511 & 11011 \\
\hline $\mathbf{2 6}$ & & 286 & 676 & 1326 & 2626 & 6526 & 13026 & 26026 \\
\hline $\mathbf{5 1}$ & & 561 & 1326 & 2601 & 5151 & 12801 & 25551 & 51051 \\
\hline $\mathbf{1 0 1}$ & 1111 & 2626 & 5151 & 10201 & 25351 & 50601 & 101101 \\
\hline $\mathbf{2 5 1}$ & 2761 & 6526 & 12801 & 25351 & 63001 & 125751 & 251251 \\
\hline $\mathbf{5 0 1}$ & 5511 & 13026 & 25551 & 50601 & 125751 & 251001 & 501501 \\
\hline $\mathbf{1 0 0 1}$ & 11011 & 26026 & 51051 & 101101 & 251251 & 501501 & 1002001 \\
\hline
\end{tabular}




\subsection{Duffing-Homes oscillator}

In case of the Duffing-Holmes oscillator set $[0,1]$ was given for both bifurcation parameters. The calculation times as the resolution of $\gamma$ is varied can be seen in Figure 9.
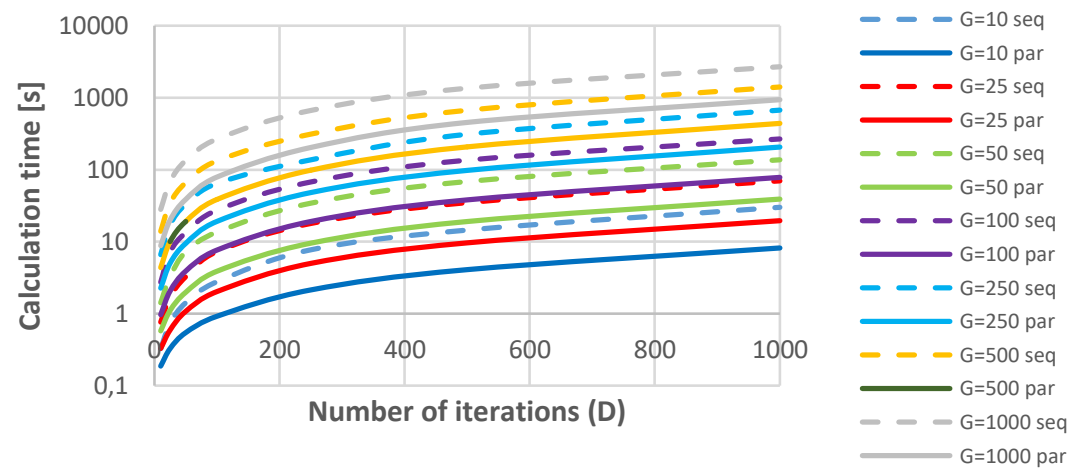

Figure 9. Calculation times versus the resolution (different resolution for $G$ ) in case of the Duffing-Holmes oscillator

It can be seen that the calculation time increases as the resolution is increased. The calculation time of the parallel algorithm is less than $1000 \mathrm{~s}$ at the highest resolution. The speedup and the efficiency are shown in Figure 10.

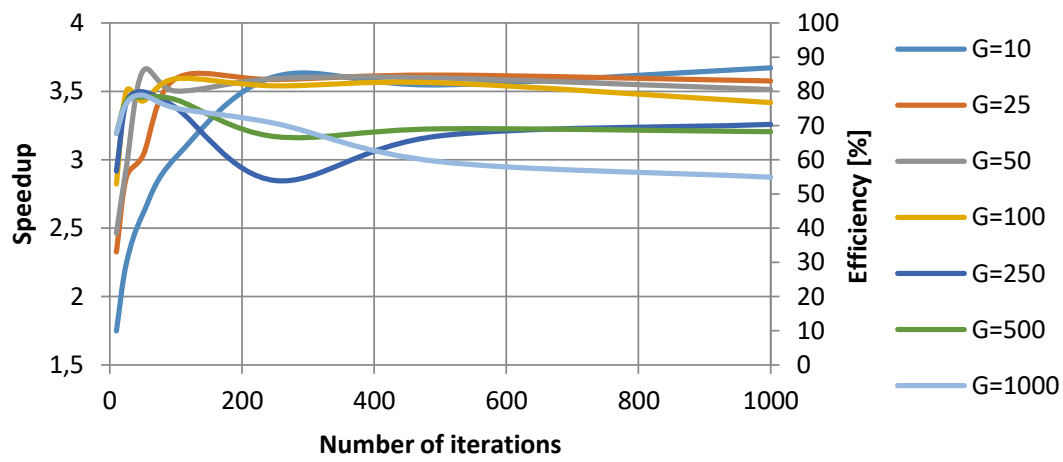

Figure 10. Speedup and efficiency versus the resolution (different resolution for $G$ ) in case of the Duffing-Holmes oscillator 
From Figure 10 the following can be derived:

- When $\mathrm{G}=10$ and $\mathrm{D}$ is small $\mathrm{S}<2$ and $\mathrm{E}<50 \%$. When $\mathrm{G}$ is increased both of them increases. When $\mathrm{D}=1000 \mathrm{~S}>3.6$ and $\mathrm{E}>91 \%$. It is the best result achieved.

- When $\mathrm{G}=25$ and $\mathrm{G}$ is small $\mathrm{S}<2.5$ and $\mathrm{E}<60 \%$. When $\mathrm{D}$ is increased both increases fast and when $\mathrm{D}>100$ an approximately constant $\mathrm{S}=3.58$ and $\mathrm{E}=90 \%$ can be achieved.

- When $\mathrm{G}=50$ and $\mathrm{G}$ is small $\mathrm{S}=2.5$ and $\mathrm{E}=60 \%$. When $\mathrm{D}$ is increased first there is a fast increase in $\mathrm{G}$ and $\mathrm{D}$. The best results could be achieved, when $\mathrm{D}=50$, in this case $\mathrm{S}>3.6$ and $\mathrm{E}>91 \%$. When $\mathrm{D}$ is increased further first there is a decrease in both. An average $S=3.5$ and $S=89 \%$ could be achieved.

- When $\mathrm{G}=100$ and $\mathrm{D}$ is small $\mathrm{S}=2.8$ and $\mathrm{E}=70 \%$. When $\mathrm{D}$ is increased there is an increase in both. The best result could be achieved, when $\mathrm{D}=100$. In this case $\mathrm{S}=3.6$ and $\mathrm{E}=90 \%$. When $\mathrm{D}$ is further increased both of them decreases, but $\mathrm{E}>85 \%$ remains.

- When $\mathrm{G}=250$ and $\mathrm{D}$ is small $\mathrm{S}=2.9$ and $\mathrm{E}=72 \%$. When $\mathrm{D}$ is increased both of them increases (till $\mathrm{D}=50$ ), then they decrease (till $\mathrm{D}=250$ ) and then they increase again till $\mathrm{S}=3.25$ and $\mathrm{E}=81 \%$. The best results could be achieved when $\mathrm{D}=50$ and the worst results when $\mathrm{D}=250$.

- When $\mathrm{G}=500$ and $\mathrm{D}$ is increased (till $\mathrm{D}=50$ ) $\mathrm{S}$ and $\mathrm{E}$ increase, after they decrease (till $\mathrm{D}=250$ ) and after a small increase they remain constant $(\mathrm{S}=3.22$ and $\mathrm{E}=80 \%)$.

- When $\mathrm{G}=1000$ and $\mathrm{D}$ is increased after a short increase $\mathrm{S}$ decreases from 3.5 to 2.8 and $\mathrm{E}$ decrease from $87 \%$ to $72 \%$. At this resolution lack of memory also occurred.

The overhead is shown is Figure 11. 


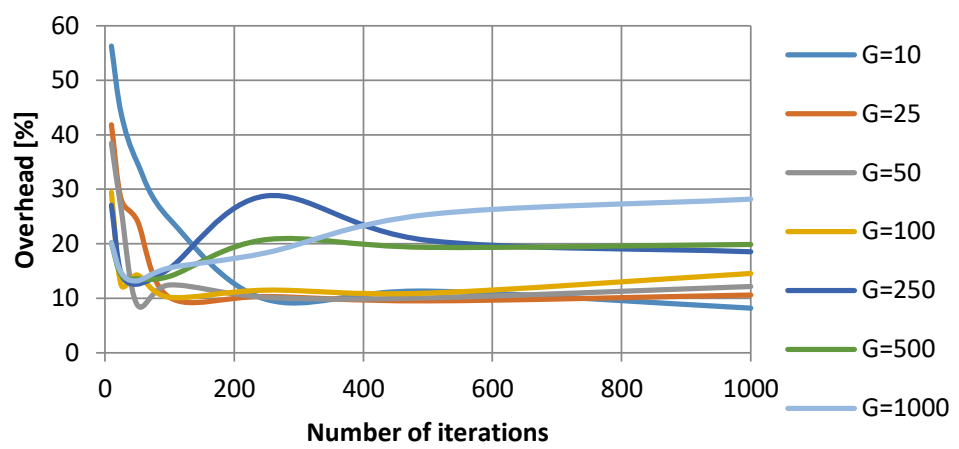

Figure 11. Overhead versus the resolution (different resolution for $G$ ) in case of the Duffing-Holmes oscillator

From Figure 11 the following can be derived:

- When the resolution is small the overhead is high (40-60\%), which means the communication time between the nodes is high compared to the useful calculation time

- The overhead increases (till almost 30\%) when $\mathrm{G}=1000$ and the resolution is increased

- The overhead is increased till $20 \%$ when $\mathrm{G}=250$ and $\mathrm{G}=500$ and the resolution is increased

- All the other cases the overhead is $10 \%$, which means only $10 \%$ of the total running time is spent on the communication

The effect of different $G$ and $D$ values in case of the same number of iterations to efficiency and overhead was also compared. The results can be seen in Figure 12. 


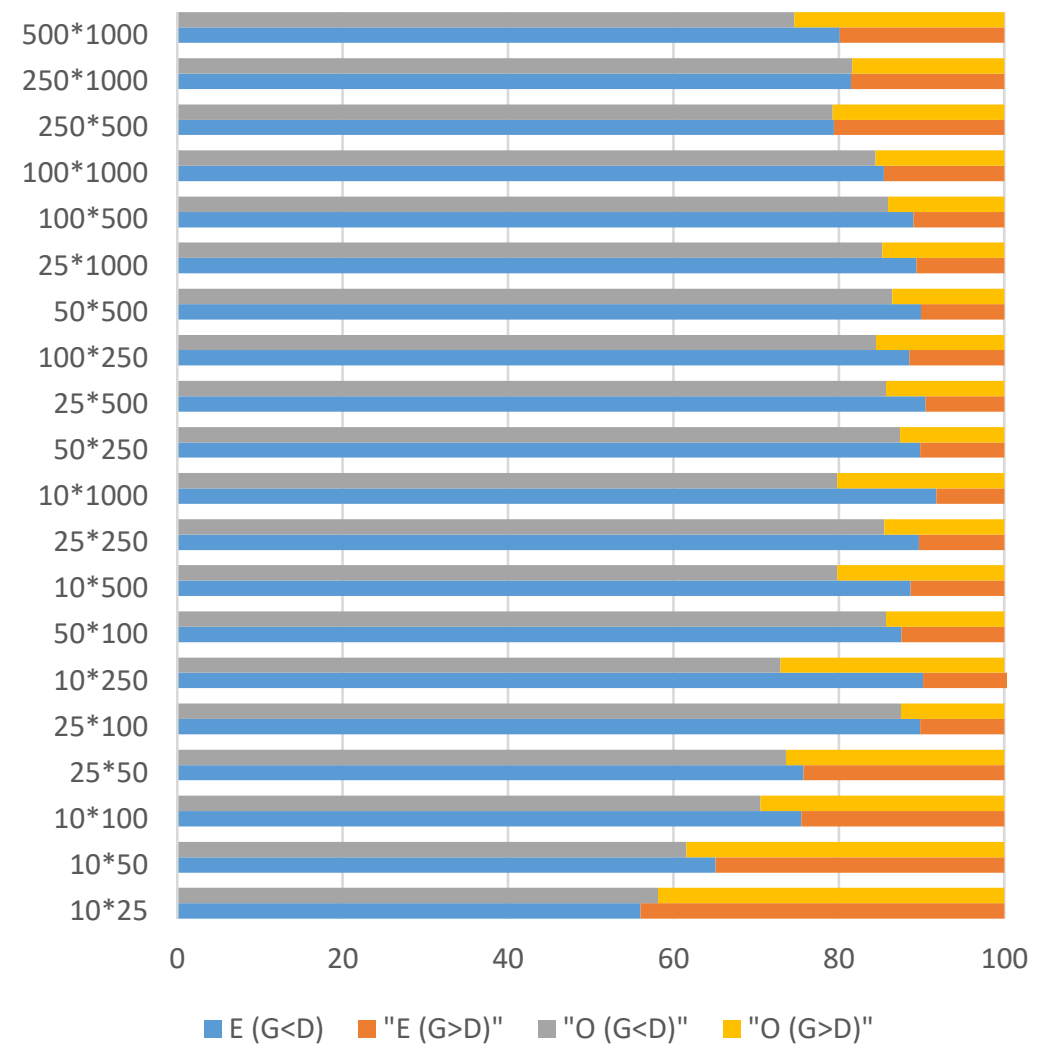

Figure 12. Comparison of efficiency and overhead with different $G$ and $D$ values at the same number of iterations in case of the Duffing-Holmes oscillator

It can be seen that except the smallest resolution the speedup and efficiency were better, when $G<D$. It means that it is more effective to calculate a lot of low resolution 2D bifurcation diagram in parallel, that to calculate a low amount of high resolution $2 \mathrm{D}$ bifurcation diagrams.

The average difference is $4 \%$. The smallest difference is at resolution $500 \times 250$ and $1000 \times 250$, it is $<20 \%$ is both cases. The difference was small $(<3 \%)$ in resolution $10 \times 25,25 \times 50,25 \times 100,50 \times 250$ and $100 \times 1000$. The maximum difference is $17.27 \%$ (means 0.69 less speedup), which is at resolution $10 \times 250$. 
The overhead is also much higher at this resolution, when $\mathrm{G}>\mathrm{D}$. The difference is also high at resolution $10 \times 500(8.84 \%)$ and $10 \times 1000(12 \%)$. It also shows that it is not efficient to calculate small number of detailed 2D bifurcation diagrams in parallel, as communication can be time consuming.

Figure 13. shows the average speedup, efficiency and overhead versus the average number of iterations.
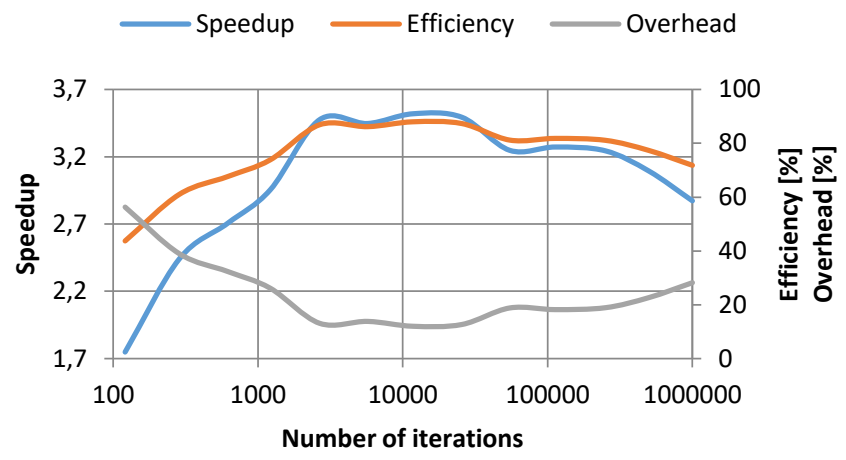

Figure 13. Average speedup, efficiency and overhead versus the average number of iterations in case of the Duffing-Holmes oscillator

It can be seen that $\mathrm{S}$ and $\mathrm{E}$ increases till the number of iterations is 12000 , after it decreases. The speedup is still $>3$ and the efficiency is $77 \%$ with 500000 iterations. The average overhead first is above $40 \%$ and when the resolution is increased (around 3000 iterations) it desreases under $20 \%$.

\subsection{Van der Pol oscillator}

For this test $A=[0,2]$ and $\mu=[0,15]$ sets were given. The calculation times as the resolution of $\mu$ is varied can be seen in Figure 14 . 

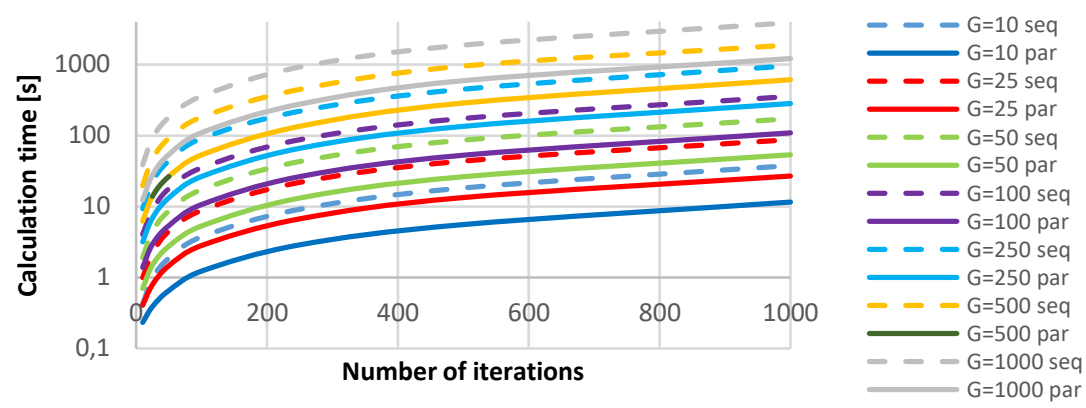

Figure 14. Calculation times versus the resolution (different resolution for $G$ ) in case of the Van der Pol oscillator

It can be seen that the calculation time increases as the resolution is increased. The calculation time of the parallel algorithm is less than $1500 \mathrm{~s}$ at the highest resolution. The speedup and the efficiency are shown in Figure 15.

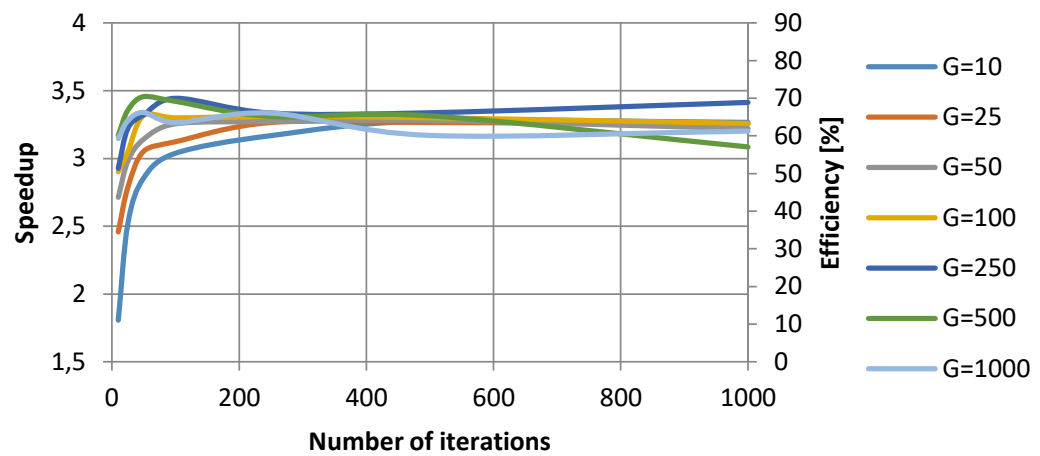

Figure 15. Speedup and efficiency versus the resolution (different resolution for $G$ ) in case of the Van der Pol oscillator

From Figure 15 the following can be derived:

- When $\mathrm{G}=10$ and $\mathrm{D}$ is small $\mathrm{S}<2$ and $\mathrm{E}<50 \%$. When $\mathrm{G}$ is increased both of them increases till $\mathrm{S}=3.2$ and $\mathrm{E}=81 \%$. 
- When $\mathrm{G}=25$ and $\mathrm{G}$ is small $\mathrm{S}=2.5$ and $\mathrm{E}=61 \%$. When $\mathrm{D}$ is increased both increases fast and when $\mathrm{D}>250$ an approximately constant $\mathrm{S}=3.2$ and $\mathrm{E}=81 \%$ can be achieved.

- When $\mathrm{G}=50$ and $\mathrm{G}$ is small $\mathrm{S}=2.7$ and $\mathrm{E}=67 \%$. When $\mathrm{D}$ is increased till 500 both of them increases till $\mathrm{S}=3.28$ and $\mathrm{E}=82 \%$. When $\mathrm{D}$ is further increased there is a small decrease in $\mathrm{E}$ and $\mathrm{S}$.

- When $\mathrm{G}=100$ and $\mathrm{D}$ is small $\mathrm{S}=2.9$ and $\mathrm{E}=72 \%$. When $\mathrm{D}$ is increased till 500 there is an increase in both till $\mathrm{S}=3.3$ and $\mathrm{E}=82 \%$. When $\mathrm{D}$ is further increased there is a small decrease in both of them.

- When $\mathrm{G}=250$ and $\mathrm{D}$ is small $\mathrm{S}=2.9$ and $\mathrm{E}=73 \%$. When $\mathrm{D}$ is increased both of them increases till $\mathrm{D}=100(\mathrm{~S}=3.4$ and $\mathrm{E}=86 \%)$. When $\mathrm{D}$ is further increased first there is a small decrease (till $\mathrm{D}=500$ ) and then a small increase in both.

- When $\mathrm{G}=500$ and $\mathrm{D}$ is increased (till $\mathrm{D}=50$ ) $\mathrm{S}$ and $\mathrm{E}$ increase. This is the best result achieved: $\mathrm{S}=3.45$ and $\mathrm{E}=86 \%$. After that they decrease till $\mathrm{S}=3.1$ and $\mathrm{E}=77 \%$.

- When $\mathrm{G}=1000$ and $\mathrm{D}$ is increased after a short increase $\mathrm{S}$ decreases from 3.3 to 3.2 and $\mathrm{E}$ decreases from $83 \%$ to $80 \%$.

The overhead is shown is Figure 16.

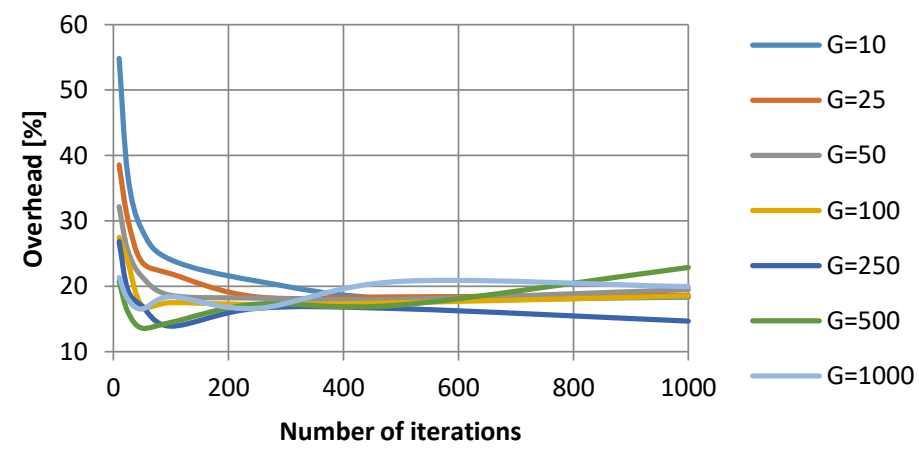

Figure 16. Overhead versus the resolution (different resolution for $G$ ) in case of the Van der Pol oscillator 
From Figure 16 the following can be derived:

- When the resolution is small the overhead is high (40-55\%), which means the communication time between the nodes is high compared to the useful calculation time

- The overhead increases (till almost $25 \%$ ) when $\mathrm{G}=500$ as the resolution is increased

- The overhead is increased above $20 \%$ when $\mathrm{G}=1000$ and the resolution is increased

- All the other cases the overhead is $<20 \%$, which means less than $20 \%$ of the total running time is spent on the communication

The effect of different $G$ and $D$ values in case of the same number of iterations to efficiency and overhead was also compared. The results can be seen in Figure 17.

It can be seen that most cases the speedup and efficiency were better (and the overhead lower), when $\mathrm{G}>\mathrm{D}$, only at some higher resolution cases were the efficiency better in case of $G<D$. It means that similarly to the Duffing-Holmes oscillator it is more efficient to create a lot of low resolution 2D diagrams, than to create a smaller number of higher resolution 2D diagrams in parallel.

The average difference is $2.3 \%$. The smallest difference is at resolution $100 \times 1000$ and it is $<0.15 \%$. The difference is less than $4 \%$ in almost all cases except resolution $250 \times 10(6.04 \%)$ and resolution $500 \times 50(4.4 \%)$. The difference in overhead is also higher at these resolutions. The difference in efficiency and overhead was much less than the difference with the Duffing-Holmes oscillator, which means that the tasks could be divided between the nodes more effectively. 
F. Hajdu-Acta Technica Jaurinensis, Vol. 11, No. 2, pp. 61-83, 2018

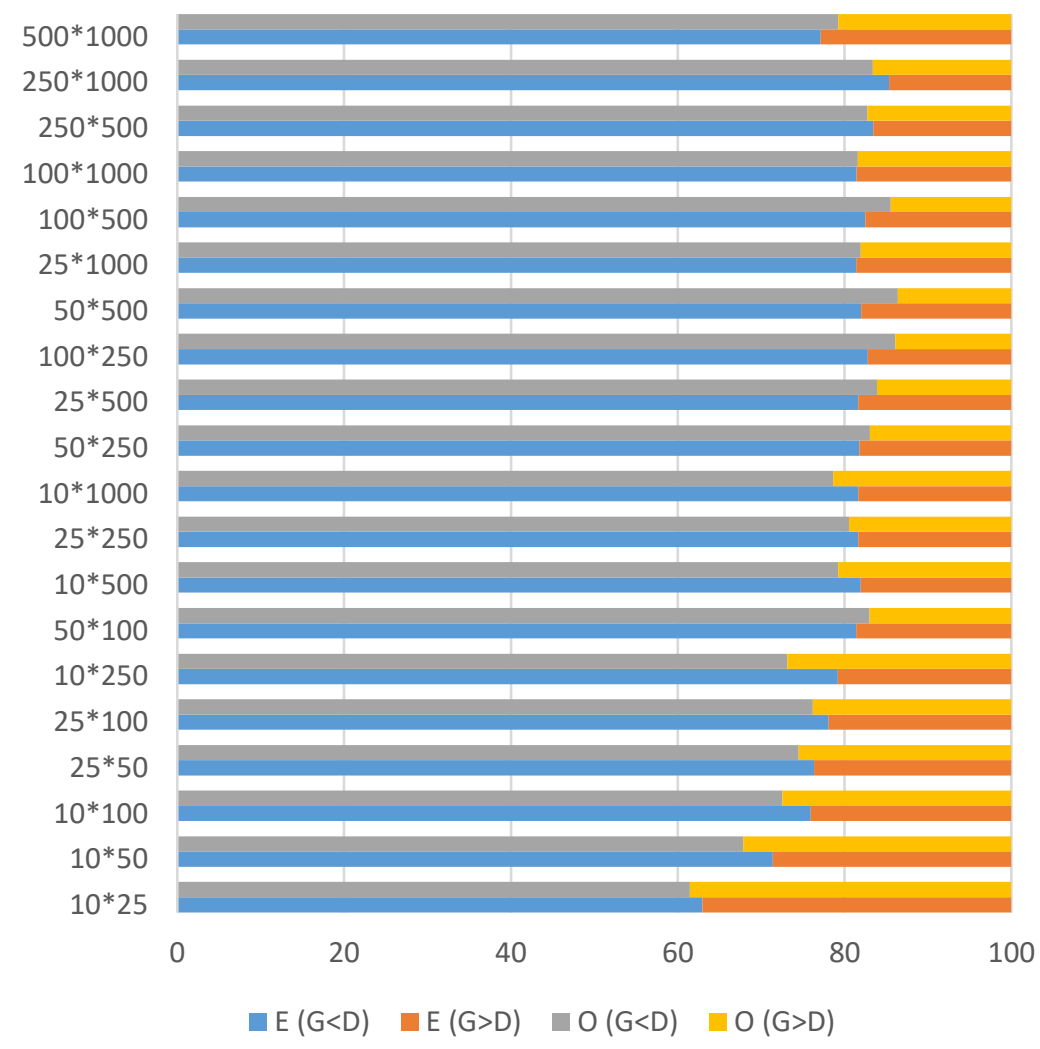

Figure 17. Comparison of efficiency and overhead with different $G$ and $D$ values at the same number of iterations in case of Van der Pol oscillator

Figure 18 shows the average speedup, efficiency and overhead versus the number of iterations. 

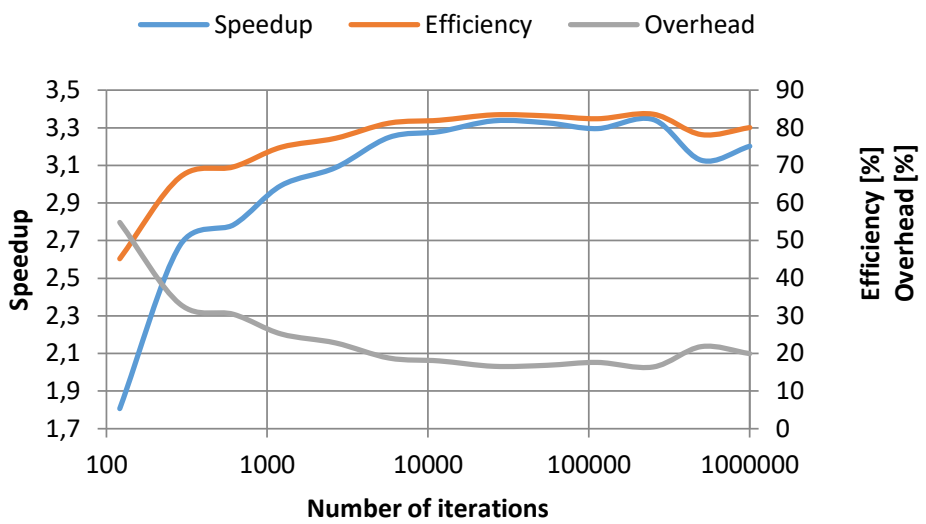

Figure 18. Average speedup, efficiency and overhead versus the number of iterations in case of the Van der Pol oscillator

It can be seen that $\mathrm{S}$ and $\mathrm{E}$ increases till the number of iterations is 25000 , after it is nearly constant $(\mathrm{S}=3.3$ and $\mathrm{E}=83 \%$ ) till number of iterations is 250000 , ather that it decreases. The speedup is still $>3.1$ and the efficiency is $78 \%$. The average overhead is first $>40 \%$ and when the resolution is increased (around 6000 iterations) it desreases under $20 \%$.

\section{Conclusions and further development}

The parallel numerical creation of a 3D bifurcation diagram of two simple nonlinear oscillators was carried out with a simple and fast iterative algorithm. An average 3-fold speedup could be achieved with average $76 \%$ efficiency in both cases. The best results could be achieved with the Duffing-Holmes oscillator, when the number of iteration was 12000 (3.5-fold speedup and 87\% efficiency). A better speedup and efficiency could be achieved with the Duffing-Holmes oscillator when the number of iteration was between 2000 and 50000 as it can be seen in Figure 19. At all other resolutions the results were better with the Van der Pol oscillator. The average overhead was high till 3000 iterations $(30-40 \%)$, then it was $<20 \%$ till 100000 iteraritons in both cases. When the resolution is further increased the average overhead increases till 20-30\% (Figure 19). 

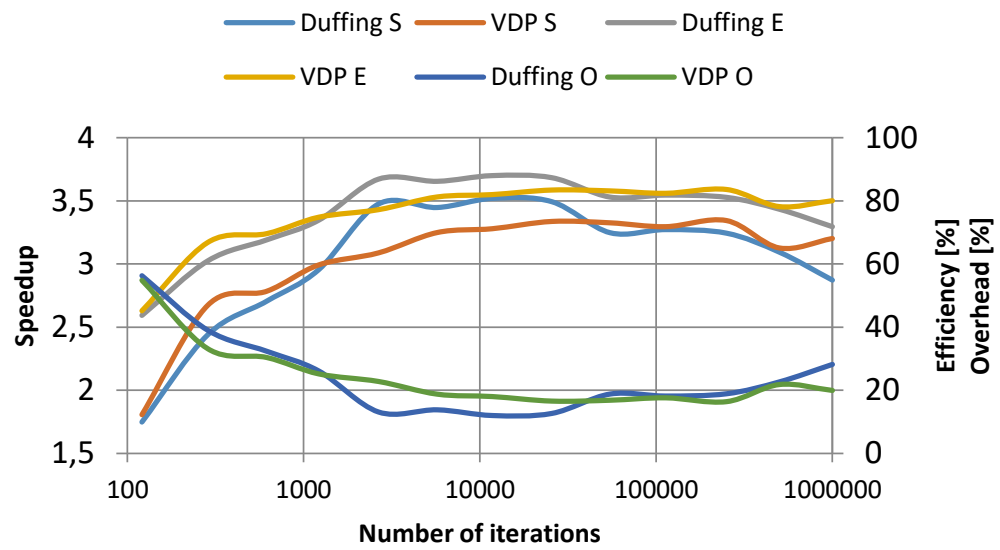

Figure 19. The average speedup, efficiency and overhead versus the number of iterations

With the presented algorithm a high resolution 3D bifurcation diagram can be created of simple systems very effectively with simple PC-s with low number of cores.

Next task is the better visualization of the results, which means using surface plot instead of point plot. Some initial experiments have already been carried out creating surface plots using Maple, but for visualization there was not enough memory.

Other task in the future is utilizing supercomputers to create even more detailed 3D bifurcation diagram of more complex systems and to create other highcalculation time diagrams, like frequency spectrum maps in parallel

\section{Acknowledgement}

The publishing of this paper was supported by the ÚNKP-17-3 New National Excellence Program of the Ministry of Human

Capacities. 


\section{References}

[1] Y.A. Kuznetsov: Elements of applied bifurcation theory, Springer, 1998.

[2] G. Stépán, R. Szalai, S. J. Hogan: The Chaotic Oscillations of Highspeed Milling, In: G. Rega, F. Vestroni (eds) IUTAM Symposium on Chaotic Dynamics and Control of Systems and Processes in Mechanics. Solid Mechanics and its Applications, vol 122. Springer, Dordrecht, 2005, pp. 147158, doi: 10.1007/1-4020-3268-4_14

[3] T. L. Schmitz, A. Honeycutt: The Extended Milling Bifurcation Diagram, Procedia Manufacturing, Vol. 1, pp. 466-474, 2015, doi: 10.1016/j.promfg.2015.09.005.

[4] H. Marzbani,M. Fard, R. Jazar: Chaotic Behavior of Hydraulic Engine Mount, Procedia Computer Science, Vol. 96, 2016, pp. 1597-1608, doi: 10.1016/j.procs.2016.08.207

[5] J. Fakhraei, H.M. Khanlo, M. Ghayour: Chaotic behaviors of a ground vehicle oscillating system with passengers, Scientia Iranica. Vol. 24. No. 3, 2015, pp. 1051-1068. doi: 10.24200/sci.2017.4088.

[6] T. Salau: Bifurcation Diagrams of Nonlinear RLC Electrical Circuits, International Journal of Science and Technology, Vol. 1 No. 3, 2011, pp. 126-139,

[7] I.Flegar, D. Pelin, D. Zacek: Bifurcation diagrams of the buck converter, 9th International Conference on Electronics, Circuits and Systems, Dubrovnik, Croatia, Paper 7562243, 2002, doi: 10.1109/ICECS.2002.1046412

[8] L. Houfek, M. Houfek, J. Krejsa, C. Kratochvíl, J. Kolácny, P. Nykodym: Bifurcation and Chaos in Electromechanical Drive Sysstems with Small MPTPRS, Engineering MECHANICS, Vol. 15 No. 5, 2008, pp. 329-336

[9] C. Kratochvíl, M. Houfek, J. Koláčný, R. Kř́ǐž, L. Houfek , J. Krejsa: Chaos in drive systems, Applied and Computational Mechanics Vol. 1, pp. 121-126,

[10] P. Simon: Differential Equations and Dynamical Systems (in Hungarian). Eötvös Loránd University, lecture notes, Budapest, Hungary, 2013 [cited 2018.01.14.], 
http://www.tankonyvtar.hu/hu/tartalom/tamop412A/2011_0025_mat_11/inde x.html

[11] D. Stiefs, E. Venturino, U. Feudel: Computing 3D Bifurcation Diagrams, AIP Conference Proceedings Vol. 1048 No. 1, 2008, doi: 10.1063/1.2991095

[12] L. Környei: Computation Techniques in a High Performance Parallel Simulation of Gas Dynamics in a Combustion Chamber. Acta Technica Jaurinensis, Vol. 4. No. 4., 2011, pp. 443-452

[13] B. W. Kooi: Numerical Bifurcation Analysis of Dynamical Systems, Faculty of Earth and Life Sciences, Department of Theoretical Biology, Vrije University, The Netherlands, lecture notes, 2009, [cited 2018.01.31], http://www.bio.vu.nl/thb/course/mri/mri_syllabus.pdf

[14] D. Stiefs, T. Gross, R. Steuer, U. Feudel: Computation and Visualization of Bifurcation Surfaces, International Journal of Bifurcation and Chaos, Vol 18. No 8, 2008, pp. 2191-2206, doi: 10.1142/S0218127408021658

[15] T. Gross, U. Feudel: Analytical Search for Bifurcation Surfaces in Parameter Space, Physica D: Nonlinear Phenomena, Vol. 195, No. 3-4, 2004, pp. 292302, doi: 10.1016/j.physd.2004.03.019

[16] J. A. Corea-Araujo, F. González-Molina, J. A. Martínez: Tools for Characterization and Assessment of Ferroresonance Using 3-D Bifurcation Diagrams, IEEE Transactions on Power Delivery, Vol. 29 No. 6, 2014, pp. 2543-2551, doi: 10.1109/TPWRD.2014.2320599

[17] G. Guerra, J. A. Corea-Araujo, J. A. Martinez-Velasco, F. González-Molina: Generation of Bifurcation Diagrams for Ferroresonance Characterization Using Parallel Computing, The European EMTP-ATP Users Group (EEUG) Conference, Grenoble, France, 2015

[18] W. Souhail, H. Khamari, M. F. Mimouni: From 2D to 3D Bifurcation Structures in Field Oriented Control of a PMSM, Journal of Electrical \& Electronic Systems, Vol 5. No 3., Paper 1000196, 2016, doi: 10.4172/23320796.1000196

[19] F. Hajdu F., Gy. Molnárka: Numerical examination of a system model with a nonlinear component, TEAM 2016: Proceedings of the 8th International 
Scientific and Expert Conference, Trnava, Slovakia, 2016.10.19-2016.10.21., 2016. pp. 12-16.

[20] G. Piedrafita, K. Ruiz-Mirazo, P.-A. Monnard, A. Cornish-Bowden, F. Montero: Viability Conditions for a Compartmentalized Protometabolic System: A Semi-Empirical Approach, PLoS ONE, Vol. 7 No. 6, Paper e39480, 2012, doi: 10.1371/journal.pone.0039480

[21] S. Lynch: Dynamical systems with applications using maple, Birkhäuser Boston, 2010.

[22] Maróti Gy. Investigating parameterized model of n-gonal wheel's motion, Pollack Periodica, Vol. 6, No. 2, 2011, pp. 25-36. doi:

10.1556/Pollack.6.2011.2.3.

[23] Perjési-Hámori I. Two dimensional mathematical model of heat-transmission using MAPLE, IFAC-PapersOnLine, Vol. 48, No. 1, 2015 pp. 689-690. doi: 10.1016/j.ifacol.2015.05.207

[24] F. Hajdu, Gy. Molnárka: Parallelization of Numerical Examination of Nonlinear Systems using Maple, in P. Iványi, B.H.V. Topping, G. Várady, (Eds) Proceedings of the Fifth International Conference on Parallel, Distributed, Grid and Cloud Computing for Engineering, Civil-Comp Press, Stirlingshire, UK, Paper 30, 2017. doi:10.4203/ccp.111.30

[25] L. Környei, G. Kallós, D. Fülep: Parallel Computations on the Blade Server at Széchenyi István University, Acta Technica Jaurinensis, Vol 3. No. 1, 2010, pp. 111-126

[26] D.E. Womble, "The challenge of massively parallel computing, Parallel Computing" in E. H. D'Hollander,G. R. Joubert, F.J. Peters, H.J. Sips (Eds) Parallel Computing: Fundamentals and Applications: Proceeding of the International Conference ParCo99, Imperial College Press, 2000, pp. 32-43, doi: 10.1142/9781848160170_0003

[27] D. Petcu: Performance Metrics for Parallel Programs, lecture notes, West University of Timisoara, Romania, 2010, [cited 2018.04.30], http://web.info.uvt.ro/ petcu/calcul/PC-2.pdf 